\title{
The Neural Crest and Cancer: A Developmental Spin on Melanoma
}

\author{
Paul M. Kulesa ${ }^{a, b}$ Jason A. Morrison ${ }^{a}$ Caleb M. Bailey ${ }^{a}$ \\ ${ }^{a}$ Stowers Institute for Medical Research, Kansas City, Mo., and ${ }^{\mathrm{b}}$ Department of Cell Biology and Anatomy, \\ University of Kansas School of Medicine, Kansas City, Kans., USA
}

\section{Key Words}

Neural crest · Melanoma - Metastasis - Chick embryo · Laser capture microdissection $\cdot$ Gene profiling $\cdot$ High resolution imaging

\begin{abstract}
Neural crest (NC) cells undergo an epithelial to mesenchymal transition (EMT) in order to exit from the dorsal neural tube. Similarly, ancestrally related melanoma cells employ an EMT-like event during the initial stages of metastasis to dissociate from surrounding keratinocytes. Whether or not the molecular pathogenesis and cellular dynamics of melanoma metastasis resemble the embryonic NC invasion program is unclear. Here, we highlight advances in our understanding
\end{abstract}

This paper is from the 5th International EMT Meeting, Singapore, October 10-13, 2011.

Further articles in this special topic series: 1. Newgreen, D.F., E.W. Thompson (2013) Progress in epithelial-mesenchymal transition research. Cells Tissues Organs 197: 421-423. 2. Said, N.A.B.M., K.J. Simpson, E.D. Williams (2013) Strategies and challenges for systematically mapping biologically significant molecular pathways regulating carcinoma epithelial-mesenchymal transition. Cells Tissues Organs 197: 424-434. 3. Vargas, D.A., O. Bates, M.H. Zaman (2013) Computational model to probe cellular mechanics during epithelial-mesenchymal transition. Cells Tissues Organs 197: 435-444. 4. Elsum, I.A., P.O. Humbert (2013) Localization, not important in all tumor-suppressing properties: a lesson learnt from Scribble. Cells Tissues Organs 198: 1-11.

\section{KARGER}

E-Mail karger@karger.com

www.karger.com/cto of tumor cell behaviors and plasticity, focusing on the relationship between melanoma and the NC invasion programs. We summarize recent discoveries of NC cell guidance and emerging in vivo imaging strategies that permit single cell resolution of fluorescently labeled tumor cells, with a focus on our recently developed in vivo chick embryo transplant model. Crucial to the molecular pathogenesis of metastasis, we highlight advances in gene profiling of small cell numbers, including our novel ability to gather gene expression information during distinct stages of melanoma invasion. Lastly, we present preliminary details of a comparison of specific genetic pathways associated with the early phases of melanoma invasion and known NC induction and migration signals. Our results suggest that malignant melanoma cells hijack portions of the NC program to promote plasticity and facilitate metastasis. In summary, there is considerable power in combining an in vivo model system with molecular analysis of gene expression, within the context of established developmental signaling pathways, to identify and study the molecular mechanisms of metastasis.

Copyright $\odot 2013$ S. Karger AG, Basel

\section{Abbreviations used in this paper}

EMT epithelial to mesenchymal transition

LCM laser capture microdissection

NC neural crest 


\section{Introduction}

Multipotent tumor cells and many embryonic progenitor cells share common characteristics of cell plasticity and invasiveness. The manner by which these cells invade peripheral tissues involves migration through many different microenvironments and interactions with numerous other cell types and structures. The signals between a multipotent cancer cell or embryonic progenitor cell and its microenvironment are thought to be complex and manifest in a combination of intrinsic and extrinsic cell-cell and cellmatrix interactions. Exciting studies have shown that signals potentially derived from the embryonic microenvironment can influence embryonic stem cells [Takahashi et al., 2007a, b; Yu et al., 2007; Hochedlinger, 2011], multipotent tumor cells [Hendrix et al., 2007; Abbott et al., 2008] and adult cell fate and plasticity [Real et al., 2006]. One of the next logical steps is to resolve the nature of the in vivo cellular and molecular mechanisms critical to the survival and programming of cell fate and invasion. Further insights from studies at the interface of developmental and tumor biology that exploit the accessibility of the embryo may help to determine the extent of the convergence of embryonic and tumorigenic signaling pathways involved in regulating cell fate and invasive ability.

Within the developing vertebrate embryo, the neural crest (NC) represents a multipotent, highly migratory cell population that produces multiple cell types to contribute to bone and cartilage of the face, function of the heart and gut, and the entire peripheral nervous system [Le Douarin and Kalcheim, 1999; Acloque et al., 2009; Zhang et al., 2010]. NC cells undergo an epithelial to mesenchymal transition (EMT) to exit the neural tube, then sort into segregated migratory streams and invade the embryo along the vertebrate axis in a programmed rostral-to-caudal manner [Kulesa and Gammill, 2010]. Signals within the neural tube and the many different microenvironments encountered by the migratory $\mathrm{NC}$ are thought to spatially permit or inhibit NC cell movements, leading to the sculpting of NC cells into particular migratory streams that reach precise peripheral targets [Gammill and Roffers-Agarwal, 2010; Kulesa et al., 2010; Kulesa and Gammill, 2010]. Time-lapse microscopy studies have revealed a complex set of NC cell migratory behaviors that include active avoidance of some areas, but directed movement and cell-cell contact through others, supporting the hypothesis that local microenvironments shape individual NC cell trajectories [Kuriyama and Mayor, 2008; Gammill and Roffers-Agarwal, 2010; Kulesa et al., 2010; Kulesa and Gammill, 2010; Mayor and Carmona-Fontaine, 2010].

Melanoma Exploits the NC Program
Technical advances in microscopy, cell labeling and molecular biology have allowed scientists to extend observations made by pioneers working at the intersection of developmental biology and cancer. Early research at this interface foresaw the importance of understanding basic cellular and molecular mechanisms involving EMT and cell migration. Novel efforts pioneered the development of cell labeling and culture techniques that extended observations of cell behaviors in 2D to in situ organ culture and helped to build the knowledge base of cell behaviors in 3D [Mintz and Illmensee, 1975; Erickson et al., 1980; Davis and Trinkaus, 1981; Bilozur and Hay, 1988]. This work helped to lay the groundwork for attempts to visualize in vivo tumor cell behaviors and their response to local microenvironmental cues.

In vivo imaging strategies of the tumor microenvironment represent an intense area of study that has benefitted from new technological advances in multiphoton microscopy, fluorescent reporters of cell cycle progression, and unique windowing techniques into the living adult mouse and chick and zebrafish embryos [Condeelis and Weissleder, 2010; Fukumura et al., 2010; Giampieri et al., 2010; Friedl and Alexander, 2011; Zhou, 2011; Zhang et al., 2012]. Thus, in vivo imaging is providing unique access to tumor cell behaviors in natural microenvironments (table 1).

Exciting investigations of tumor cell interactions within in vivo embryonic microenvironments have revealed unique insights into tumor cell behaviors [Kulesa et al., 2006; Topczewska et al., 2006; Hendrix et al., 2007; Kasemeier-Kulesa et al., 2008]. In particular, the investigation of tumor cell behaviors within the embryonic chick NC microenvironment exploits the ancestral link between the NC and cancer cell types, such as melanoma and neuroblastoma. The accessibility of novel cellular and molecular imaging strategies within embryos has inspired developmental and cancer biologists to move from descriptive observational studies to mechanistic analyses of the convergence of embryonic and tumorigenic signaling pathways. Here, we highlight advances in the observation of tumor cell behaviors and molecular profiling studied within the embryonic NC microenvironment.

\section{The Chick Embryo Transplant Model}

One of the strongest links between common mechanisms that underlie NC cell migration and cancer biology has come from the study of NC cell regulatory factors [Barrallo-Gimeno and Nieto, 2005; Betancur et al., 2010; 
Table 1. In vivo models of tumor cell behaviors

\begin{tabular}{llll}
\hline Animal model & Tumor type & Highlights/methods & Reference \\
\hline Chick embryo & $\begin{array}{l}\text { Mammalian tumor } \\
\text { xenograft }\end{array}$ & $\begin{array}{l}\text { Static and dynamic imaging of cell cycle progression } \\
\text { (CycleTrak) in migrating melanoma cells }\end{array}$ & Ridenour et al., 2012 \\
\hline Zebrafish embryo & $\begin{array}{l}\text { Mammalian tumor } \\
\text { xenograft }\end{array}$ & $\begin{array}{l}\text { Dynamic imaging of tumor neovascularization and evaluation } \\
\text { of anti-angiogenic compounds }\end{array}$ & Zhao et al., 2011 \\
\hline Mouse & $\begin{array}{l}\text { Orthotopic mammalian } \\
\text { tumor xenograft }\end{array}$ & $\begin{array}{l}\text { Mammary imaging window combined with photoswitchable } \\
\text { fluorescent label provides extended intravital imaging }\end{array}$ & Kedrin et al., 2008 \\
\hline Mouse & $\begin{array}{l}\text { Mammalian tumor } \\
\text { xenograft }\end{array}$ & $\begin{array}{l}\text { Skin fold chamber provides orthotopic intravital imaging } \\
\text { of early stages of tumor invasion }\end{array}$ & $\begin{array}{l}\text { Alexander et al., } \\
\text { 2008 }\end{array}$ \\
\hline Mouse & $\begin{array}{l}\text { Mammalian tumor } \\
\text { xenograft }\end{array}$ & $\begin{array}{l}\text { Simultaneous imaging of multiple fluorescent markers and } \\
\text { collagen using multi-photon microscopy }\end{array}$ & Sahai et al., 2005 \\
\hline Rat & Allograft & Skin flap surgery to expose tumor for timelapse imaging & Wyckoff et al., 2000 \\
\hline
\end{tabular}

Haldin and LaBonne, 2010; Nieto, 2011]. Work on Snail family genes expressed in early emerging NC cells has revealed that a cell's acquisition of migratory properties is partly due to repressing E-cadherin transcription during development [Cano et al., 2000; Bolos et al., 2003]. Studies of cell invasion in ductal carcinomas have discovered a role for SNAI1 in the metastatic cascade, thus highlighting its function and potential as an early biomarker for tumor metastatic potential [Blanco et al., 2002]. These studies suggest there is a great deal to be learned from the examination of embryonic signals guiding cell migration and their potential ability to regulate tumor cell invasion. Thus, the accessible chick embryonic NC cell microenvironment provides fertile ground to search for molecular signals common to the NC cell migratory program and cancer cell plasticity and invasion.

The development of in vivo models to study embryonic and tumor cell behaviors has a rich history. Mintz and Illmensee [1975] investigated the concept that the mouse embryo was accessible to transplantation of tumor cells and found that signals within the embryonic microenvironment could reprogram the tumor cells to a less destructive fate. When the hypothesis of multipotent tumor cell reprogramming was investigated more recently in the zebrafish embryo, one of the results surprisingly showed that transplanted highly aggressive human melanoma cells induced zebrafish progenitor cells to form a secondary axis [Topczewska et al., 2006]. Further investigation revealed that the aggressive melanoma cells secreted Nodal (a potent embryonic morphogen), responsible for the ectopic induction of the embryonic axis [Topczewska et al., 2006]. Thus, although the zebrafish embryo is extremely useful as a biosensor for tumor cell sig- nals [Topczewska et al., 2006; Stewart et al., 2010; Zhang et al., 2012], one of the major limitations of this system is the lack of surgical accessibility to manipulate or transplant tissue at various developmental stages.

The avian embryo has emerged as a useful tool for analyzing both NC and tumor cell interactions, providing imaging and surgical accessibility to manipulate the NC cell migratory pathways and monitor transplanted tumor cells (fig. 1). One of the major results of these types of studies occurred as early as the 1950's, when tissue transplantation experiments that placed mouse sarcoma 180 cells into the chick limb bud caused NC-derived sympathetic nerve fibers to grow out and innervate the transplanted cells [Levi-Montalcini, 1952]. Investigation of the tumor cell and nerve fiber interactions led to the discovery of nerve growth factor as the secreted attractive signal from the sarcoma 180 cells [Levi-Montalcini, 1952].

If transplanted tumor cells can influence cell movements in the host embryo, the question arises as to the extent the host cell migratory pathways can influence other migratory cell types. Early studies that investigated the influence of the chick embryonic NC microenvironment employed transplantation of a variety of migratory cell types into the avian trunk NC cell migratory pathway [Erickson et al., 1980]. When transplanted sarcoma 180 cells were analyzed after embryo reincubation, the cells were distributed along normal trunk NC pathways and usually seen as individual cells; fibroblasts, however, remained at the transplant site [Erickson et al., 1980]. More recent work supports the hypothesis that adult tumor cells can travel along host embryo NC cell migratory pathways. Injection of human SK-Mel-28 (melanoma) cells or trans-
Kulesa/Morrison/Bailey 


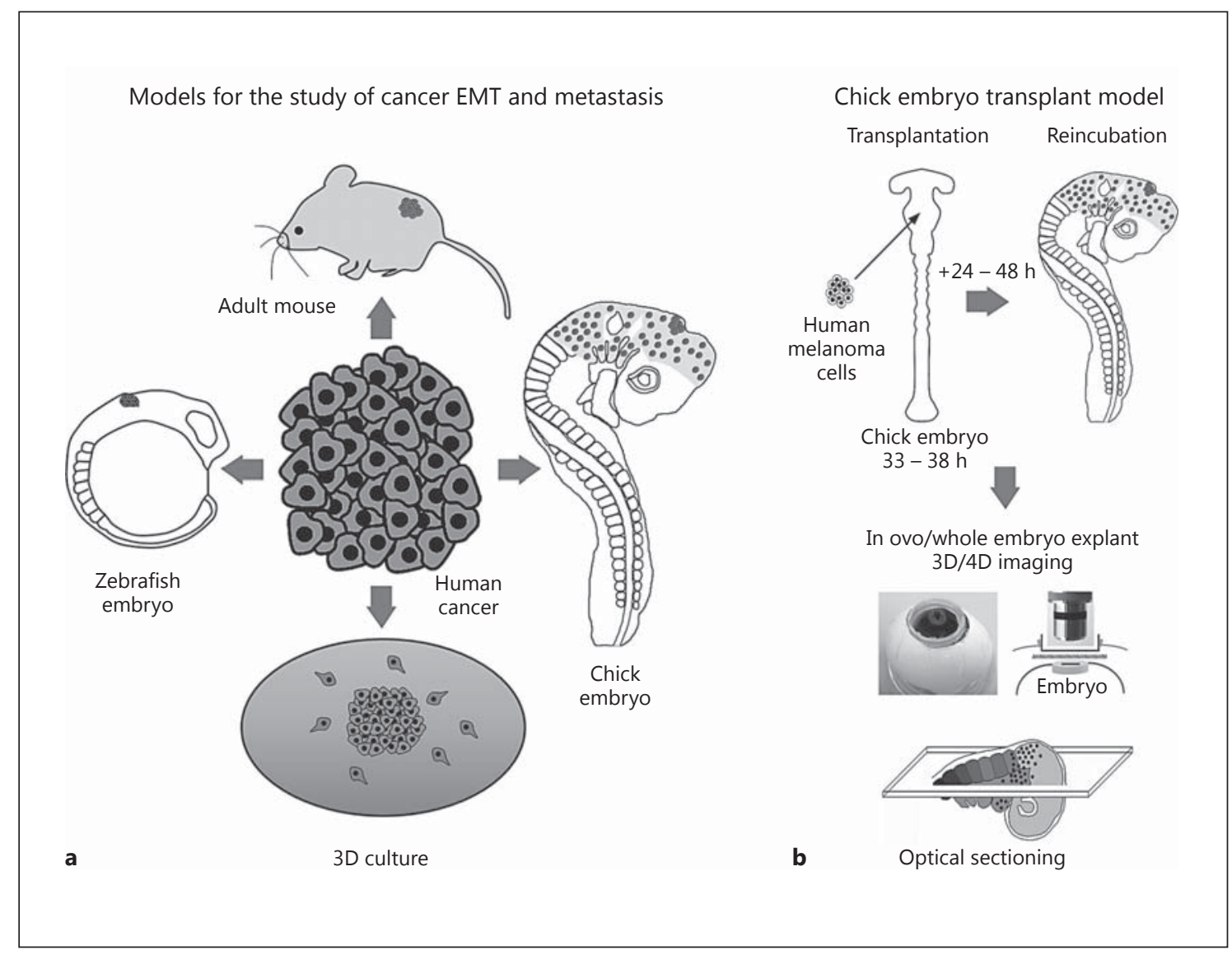

Fig. 1. Models for the study of cancer EMT and metastasis, including the Chick Embryo Transplant Model. a There are at least four model systems to analyze human tumor cell behaviors including in vitro culture, zebrafish and chick embryos, and adult mice. b The chick embryo transplant model permits transplantation of human tumor cells into the NC microenvironment and visualization of cell behaviors in vivo in $3 \mathrm{D}$ using a Teflon window into the egg that allows oxygen transfer to the embryo. plantation of C8161 aggressive melanoma cells into the chick embryo neural tube revealed that the tumor cells invaded the embryo along host NC cell cranial (summarized in fig. 2) and trunk migratory pathways [Schriek et al., 2005], and did not form tumors [Kulesa et al., 2006]. In contrast, when B16 mouse melanoma cells were injected into non-NC cell microenvironments of the chick embryo, including the eye cup, the tumor cells formed melanomas [Oppitz et al., 2007].

Imaging strategies have begun to reveal an amazing complexity of melanoma and embryonic NC cell behaviors when visualized in vivo within developmental microenvironments [Kulesa et al., 2006; Kasemeier-Kulesa et al., 2008]. Interestingly, 3D confocal analysis of GFP-labeled C8161 aggressive melanoma cells within the chick embryo NC cell microenvironment revealed that tumor cells resembled host $\mathrm{NC}$ cell morphologies, including long filopodial extensions that contacted neighboring cells and formed follow-the-leader chain-like arrays [Kulesa et al., 2006]. When we investigated the positions of transplanted invading melanoma cells our results showed that the tumor cells avoided typical NC cell-free zones and did not require a host NC cell scaffold to invade [Kulesa et al., 2006]. Also, the invading melanoma cells reached distant NC cell target sites in the head branchial arches and trunk sympathetic and dorsal root ganglia. This suggests that the chick embryonic NC microenvironment has a strong influence on adult tumor cell behaviors with an ancestral link to the NC. However, the extent to which the chick embryonic NC microenvironment influences the molecular invasion program of metastatic melanoma cells is still unclear, due in part to the challenge of analyzing changes in gene expression in small cell numbers. 
Table 2. Methods for profiling small numbers of cells

\begin{tabular}{llll}
\hline Method & Advantages & Disadvantages & References \\
\hline Single cell qPCR & Single cell required & Variance between cells, noise & $\begin{array}{c}\text { Taniguchi et al., 2009 } \\
\text { Narsinh et al., 2011 }\end{array}$ \\
\hline Single cell RNAseq & $\begin{array}{l}\text { Single cell required } \\
\text { Transcriptome analysis }\end{array}$ & Variance between cells, noise & Tang et al., 2009 \\
\hline Gene-specific PreAmp & $\begin{array}{l}\text { Small sample required, pre-validated } \\
\text { and linear amplification }\end{array}$ & Analysis of only 100 transcripts & Noutsias et al., 2008 \\
\hline $\begin{array}{l}\text { Eberwine T7 RNA } \\
\text { amplification }\end{array}$ & Transcriptome analysis & Potential bias & Van Gelder et al., 1990 \\
& & & Duftner et al., 2008 \\
& & Boelens et al., 2007 & Chen et al., 2011 \\
RNAseq & Transcriptome analysis & & Ozsolak et al., 2010 \\
& & & Tariq et al., 2011 \\
\hline
\end{tabular}

\section{Development of Gene Profiling for Small Cell Numbers}

Whole transcriptome profiling approaches such as RNAseq and microarray produce a wealth of information, but require large $(>100 \mathrm{pg})$ sample sizes [Ozsolak et al., 2010; Chen et al., 2011; Tariq et al., 2011]. In many instances, such as our transplantation of approximately 300 metastatic melanoma cells into the chick embryonic NC microenvironment, obtaining such a large amount of starting material from subpopulations of cells is not feasible. When sample size is limited, there are more directed analyses available to probe relevant genes. We describe recent advances in linear preamplification methods that have increased the number of genes that can be robustly analyzed from small amounts of starting material. We highlight application of this technology and its modifications to profile gene expression in metastatic melanoma cells transplanted into the chick embryo model and extracted at distinct temporal and spatial points of invasion.

In contrast to whole transcriptome profiling approaches, there is recent evidence of profiling single cells to highlight the variability within heterogeneous populations [Narsinh et al., 2011]. Profiling many single cells is commonly used to elucidate the range of expression within a population, but suffers from the increased number of cells required to produce accurate expression ranges. Another limitation is the soaring number of total reactions necessary for this broad type of analysis. We suggest that samples containing 10-50 cells strike a good balance between the sensitivity necessary to detect even the lowest

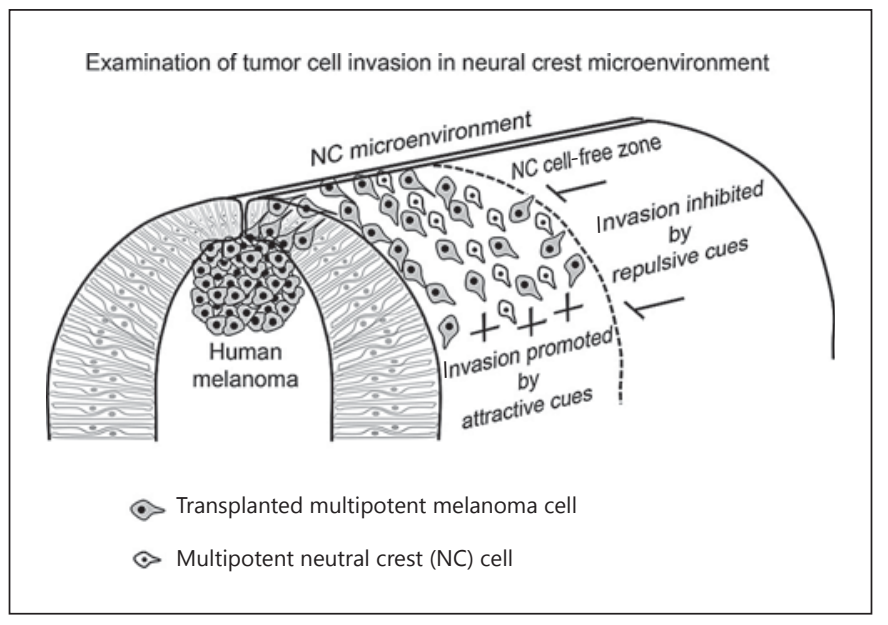

Fig. 2. Examination of tumor cell invasion in the NC microenvironment. Human metastatic melanoma cells transplanted into the chick embryonic NC microenvironment follow host migratory pathways and avoid NC cell-free zones, suggesting their invasion pattern may be influenced by NC microenvironmental signals.

expressed targets and the averaging inherent in larger populations of cells.

Several amplification protocols have been developed for expression profiling from small numbers of cells (table 2). Many kits now eliminate the need for RNA purification, a step where template is commonly lost. Ambion's Cells-to-Ct kit linearly amplifies cDNA using the same Taqman Gene Expression Assays employed during downstream RT-qPCR. A major advantage of ABI's Taqman Gene Expression Assays is that each gene-specific
16

Cells Tissues Organs 2013;198:12-21 DOI: $10.1159 / 000348418$
Kulesa/Morrison/Bailey 
Fig. 3. The combination of an in vivo model system and LCM-assisted gene profiling facilitates the identification of novel genetic interactions and putative signaling pathways. a Small numbers of cells were harvested from four distinct microenvironments: in vitro culture, the chick embryo dorsal neural tube (NT; primary transplant site) and two phases of migration, trailing and lead. Cells were collected from embryonic tissue by laser capture microdissection and samples were analyzed by RT-qPCR. b Gene expression patterns for the pluripotency markers NANOG, NES, POU5F1 and SOX2, along with the vascular endothelial cadherin $\mathrm{CDH} 5$, show a high degree of correlation. Gene expression patterns were derived from samples taken from the four microenvironments described above. $r$ values were determined using Pearson correlation.

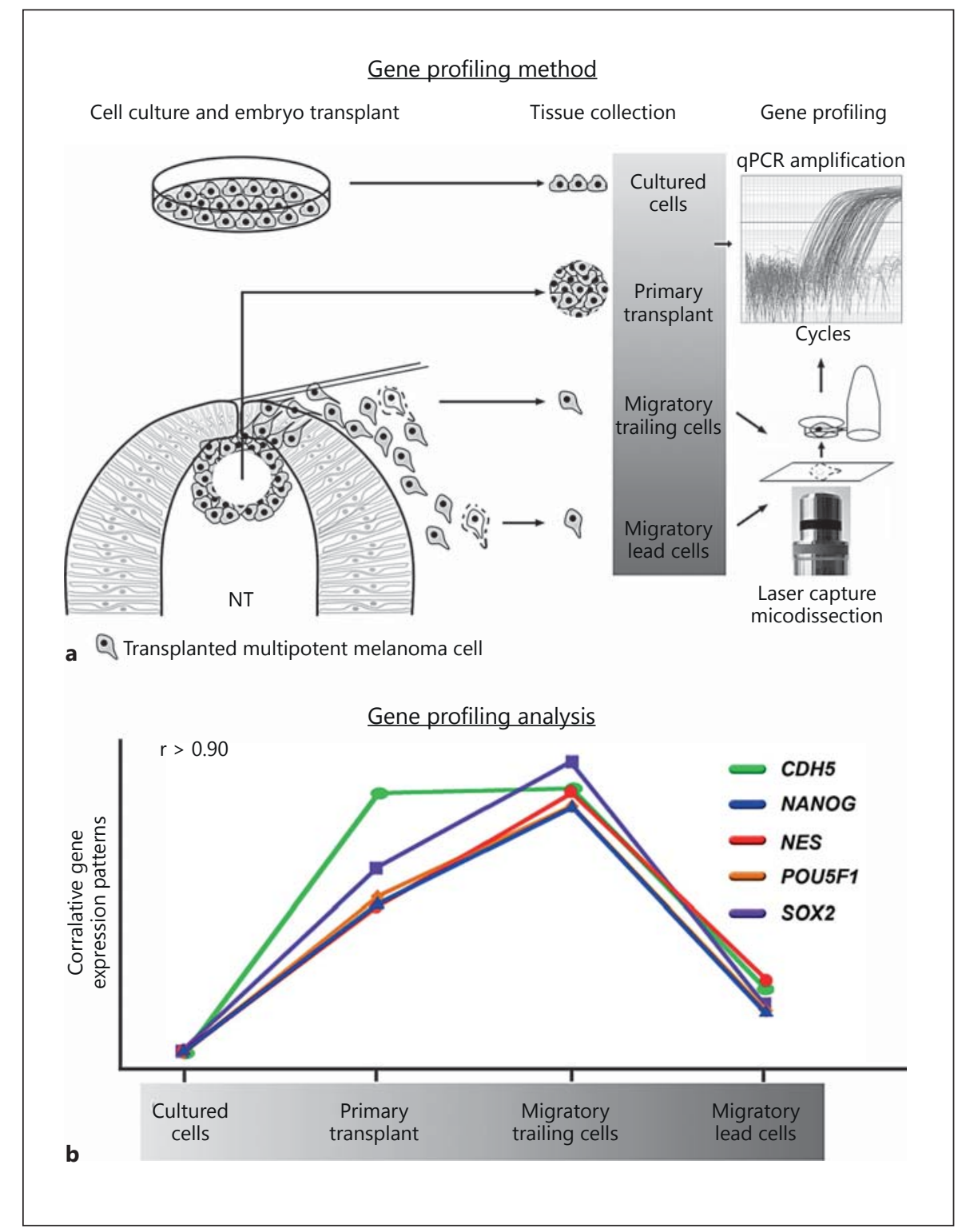

primer set is prevalidated, a process which requires substantial time and resources. The concentration at which Taqman Assays are provided currently limits the total number of genes that can be preamplified per reaction for downstream RT-qPCR.

Laser capture microdissection (LCM) is a definitive method for precisely isolating single or small subpopulations of cells from complex tissue [Emmert-Buck et al., 1996; Simone et al., 1998]. This microscopy-based technique allows visualization of target tissue and harvested samples for complete confidence during sample collection. Tissue is prepared for LCM by flash freezing, essen- tially halting all cellular processes at the moment of tissue harvest, and cryosectioning to a single-cell thickness to avoid capture of overlapping, unwanted material. Compared with FACs purification, samples harvested by LCM better represent their in vivo biological context because they do not undergo enzymatic treatment or removal from their native microenvironment prior to isolation. Furthermore, FACs isolation is less efficient with smaller sample sizes, requiring a number of cells for calibration before isolation can begin.

We have combined tissue harvest by LCM with miniaturization of a gene-specific preamplification and mi- 
crofluidic RT-qPCR on Fluidigm's BioMark system to maximize the expression information gathered from small numbers of cells without sacrificing sensitivity (fig. 3). Miniaturization of the RT-qPCR reaction on a dynamic array system requires less template and consumables per reaction, concurrently reducing the cost per RTqPCR reaction and allowing more genes to be analyzed per sample. Proper analysis of raw expression profiling data is crucial to accurate interpretation of the data. We employ complex commercially available analysis software packages that feature data quality control measures, error propagation algorithms and endogenous control reference gene stability score calculation along with the field standard delta-delta Ct method to accurately and reliably calculate relative quantities from raw expression data. Integrated downstream statistical analysis functions rapidly provide false discovery rate and confidence levels for all fold-change data.

\section{Gene Profiling of Human Melanoma after Transplantation into the Chick Embryo}

As stated above, one of the significant advantages of the chick embryo model system is access to the developing embryo, both for imaging and for molecular analysis. Using our LCM-assisted gene profiling strategy, we have interrogated gene expression profiles in human melanoma cells transplanted into the chick embryonic NC microenvironment [Bailey et al., 2012]. Because we hypothesize that metastatic melanoma cells exploit their ancestral relationship to the embryonic NC by hijacking NC genes associated with increased invasiveness and plasticity, we specifically examined a panel of genes postulated to play important roles in the NC developmental program. Small clusters (containing roughly 300 cells) of aggressive human melanoma cells (C8161) were transplanted into the dorsal neural tube at rhombomere 4 of Hamburger and Hamilton stage 9-10 chick embryos in ovo [Hamburger and Hamilton, 1951]. This stage corresponds to the delamination and emigration of host NC cells from the hindbrain. Following transplantation, embryos were incubated for $24 \mathrm{~h}$, and consequently harvested and prepared for LCM and subsequent gene expression analysis by RT-qPCR. The use of laser-assisted microdissection uniquely allowed us to capture both migrating and nonmigrating melanoma cells at the same axial level with single cell resolution, and assay these cells for changes in gene expression resulting from altering microenvironments within the developing chick embryo.
To demonstrate the validity and versatility of our system, we examined the expression of CDH5 (VE-Cadherin) in transplanted melanoma cells. It was previously demonstrated that C8161 melanoma cells aberrantly express $\mathrm{CDH} 5$ and that VE-cadherin may play an important role in the metastatic process [Hendrix et al., 2001]. However, the mechanism through which aggressive melanoma cells aberrantly express CHD5 is unknown. In our model system, $\mathrm{CDH} 5$ is induced in C8161 cells following transplantation. Further induction is observed as cells delaminate from the primary transplant and exit the dorsal neural tube. However, $\mathrm{CDH} 5$ expression begins to regress as cells migrate away from the neural tube (fig. 3). Importantly, a comparison of genes whose expression patterns correlate with the expression pattern observed for CHD5 revealed four genes with high correlation: NANOG, NES, POU5F1 and SOX2 (fig. 3). These four genes are common markers of dedifferentiation and pluripotency. Like $\mathrm{CDH} 5$, these four genes exhibited exclusive downregulation in lead migratory cells compared to trailing cells within close proximity to the neural tube. These changes to gene expression are likely the result of distinct microenvironmental cues and would not be observed in vitro. Furthermore, the high correlation observed for the regulation of these genes suggests a mechanistic link between dedifferentiation and VE-cadherin expression.

Importantly, the downregulation observed in NANOG, NES, POU5F1 and SOX2 in migrating melanoma cells succeeded the upregulation of these markers initially observed following transplantation into the lumen of the neural tube. This suggests that the embryonic microenvironment within the neural tube promotes a dedifferentiated state. Furthermore, the downregulation of plasticity genes was most evident when directly comparing lead migratory cells versus trailing cells that had recently exited the neural tube. This suggests that dedifferentiation may facilitate exit from the neural tube in a manner analogous to NC delamination, for which EMT is a requisite step [Taneyhill et al., 2007]. Then, as cells begin to migrate away from the neural tube, the loss of potent signals promoting stemness is manifest in the reduction of pluripotency markers.

Notably, mesenchymal tissue through which NC cells emigrate may also promote differentiation [Derby, 1982; Derby and Newgreen, 1982]. Likewise, as melanoma cells invade along NC routes, they may also be exposed to powerful differentiation cues, reflected by a significant decrease in the expression of pluripotency markers. This is supported by our previous findings demonstrating the reexpression of Melan A in a subset of migratory C8161
18

Cells Tissues Organs 2013;198:12-21 DOI: $10.1159 / 000348418$
Kulesa/Morrison/Bailey 
cells within our model system. Under normal culture conditions, C8161 cells are negative for Melan A gene expression. Thus, the reexpression of this marker of melanocyte differentiation suggests reprogramming toward a more differentiated state [Kulesa et al., 2006]. Indeed, the ability of melanoma cells to alter their differentiation status in concert with cell invasion has been recently documented [Giampieri et al., 2010]. Taken together, these results clearly demonstrate the dynamic regulation of gene expression with respect to changing microenvironmental signals and support the hypothesis that melanoma invasion and metastasis are facilitated by their heightened ability to sense and respond to changing microenvironments.

\section{Perspectives}

The microenvironment exerts control over the genotype and phenotype of both normal and cancer cells and plays a crucial role in the determination of cell plasticity and invasion. Clues for new therapeutic strategies may come from studies of this relationship. Yet, what is particularly challenging to the development of effective therapies is the plasticity of tumor cells. That is, tumor cell plasticity suggests high adaptivity and may represent the difference in a negative versus positive outcome.

Compelling evidence presented in the literature detailing the molecular signature of multipotent tumor cells, as well as embryonic stem and progenitor cells, demonstrate the underlying plasticity of these cell types. For example, comparative global gene analyses of aggressive and poorly aggressive human melanoma cell lines have revealed that the aggressive tumor cells express genes (and proteins) associated with multiple cellular phenotypes (including progenitor and stem cells), while simultaneously downregulating genes specific to their parental melanocytic lineage [Postovit et al., 2007]. Particularly noteworthy is evidence that molecules that play a role in embryonic NC cell guidance have common expression in highly aggressive and nonaggressive tumor cells [Kasemeier-Kulesa et al., 2008]. Aggressive melanoma cells upregulate a subset of both NC cell guidance and differentiation markers compared to their nonaggressive counterpart [Kasemeier-Kulesa et al., 2008]. Also, some aggressive melanoma cells show a significant downregulation in pigmentation pathway-associated genes, and this dedifferentiated phenotype has been correlated with a poor clinical outcome [Hendrix et al., 2007]. Therefore, it is tempting to speculate that a viable strategy for redif-

Melanoma Exploits the NC Program ferentiating the aggressive, plastic melanoma cell phenotype back to a melanocyte-like cell might lead to the suppression of metastasis.

New technology advances in in vivo imaging and gene profiling provided three significant advantages of analyzing human melanoma cells transplanted into an embryonic chick model. First, in vivo dynamic imaging using multiphoton microscopy of transplanted clumps of melanoma cells allowed single cell resolution of cell behaviors during the cell sorting within and separation from the transplanted cell cluster, as well as invasion into the chick embryonic NC microenvironment. Second, LCM and RT-qPCR permitted the extraction of single melanoma cells and gene expression readout of phenotypically matched subpopulations during the progressive stages of metastasis into the chick embryonic NC microenvironment. Recent advances in epigenetic profiling suggest small numbers of LCM-harvested cells may also be amenable to DNA methylation analysis by bisulfite sequencing [Paliwal et al., 2010]. Third, the direct comparison of $\mathrm{NC}$ and tumorigenic genes in a spatio-temporal manner allowed us to build a progressive picture of the dynamics of melanoma invasion.

Our studies provide insights into how a melanoma cell's gene expression profile changes as a system. Highly correlative changes in gene expression observed during the course of tumor cell invasion offer the potential for identification of novel genetic interactions and signaling pathways. For example, our data demonstrated the dynamic, correlative regulation of pluripotency markers with relation to the proximity to the embryonic neural tube. Interestingly, it has been recently shown that melanoma cells taken from both primary and metastatic lesions, and then grown as spheroid cultures under NC cell conditions, displayed a NC cell transcriptional signature associated with high transdifferentiation capacity and similar enhanced expression of pluripotency genes examined in our study [Ramgolam et al., 2011]. Importantly, although melanoma spheroid cultures displayed higher cell plasticity, these cells did not appear to revert to a NC stem cell state [Ramgolam et al., 2011]. These data support the hypothesis that signals within the chick neural tube may prime melanoma cell plasticity and invasiveness, and that these conditions may be partially mimicked by NC culture conditions. These findings strengthen the possibility of identifying potent embryonic signals that can regulate the tumor cell phenotype.

In summary, the concept of embryonic models to work at the interface of development and cancer has ancient roots. But, when combined with new technology advanc- 
es, these models provide multiscale information on how the cancer cell behaves in a foreign microenvironment and have the potential for exciting discoveries of the boundaries of tumor cell plasticity and invasion. This increased wealth of information about tumor cell behaviors is exciting and at the same time incredibly challenging to interpret. Traditional gain- and loss-of-function studies may need to adapt from single gene to multiple gene perturbation to effectively analyze the system. Multispectral imaging approaches will need to also adapt to allow visualization of multiple gene perturbations simultaneously, highlighted by distinct fluorescent reporters. Lastly, a major challenge will be how to translate insights gained from tumor cell behaviors within the embryonic microenvironment to the human tumor microenvironment.

\section{Acknowledgements}

We gratefully acknowledge the laboratory of Dr. Mary Hendrix for providing the C8161 melanoma cell lines used in these experiments and for thoughtful and constructive discussion. C.M.B. thanks the National Institutes of Health Ruth L. Kirschtein Postdoctoral Fellowship Program (Award No. F32CA144297) for funding and partial support from the Stowers Institute for Medical Research. P.M.K. thanks the kind generosity of the Stowers Institute for Medical Research for funding and the American Association of Anatomists Travel Bursary for partial support to attend the TEMTIA-V meeting.

\section{References}

Abbott, D.E., C.M. Bailey, L.M. Postovit, E.A. Seftor, N. Margaryan, R.E. Seftor, M.J. Hendrix (2008) The epigenetic influence of tumor and embryonic microenvironments: how different are they? Cancer Microenviron 1: 13-21.

Acloque, H., M.S. Adams, K. Fishwick, M. Bronner-Fraser, M.A. Nieto (2009) Epithelialmesenchymal transitions: the importance of changing cell state in development and disease. J Clin Invest 119: 1438-1449.

-Alexander, S., G.E. Koehl, M. Hirschberg, E.K. Geissler, P. Friedl (2008) Dynamic imaging of cancer growth and invasion: a modified skinfold chamber model. Histochem Cell Biol 130: 1147-1154.

Bailey, C.M., J.A. Morrison, P.M. Kulesa (2012) Melanoma revives an embryonic migration program to promote plasticity and invasion. Pigment Cell Melanoma Res 25: 573-583.

-Barrallo-Gimeno, A., M.A. Nieto (2005) The Snail genes as inducers of cell movement and survival: implications in development and cancer. Development 132: 3151-3161.

-Betancur, P., M. Bronner-Fraser, T. Sauka-Spengler (2010) Assembling neural crest regulatory circuits into a gene regulatory network. Annu Rev Cell Dev Biol 26: 581-603.

Bilozur, M.E., E.D. Hay (1988) Neural crest migration in 3D extracellular matrix utilizes laminin, fibronectin, or collagen. Dev Biol 125: 19-33.

-Blanco, M.J., G. Moreno-Bueno, D. Sarrio, A. Locascio, A. Cano, J. Palacios, M.A. Nieto (2002) Correlation of Snail expression with histological grade and lymph node status in breast carcinomas. Oncogene 21: 3241-3246.

Boelens, M.C., G.J. te Meerman, J.H. Gibcus, T. Blokzijl, H.M. Boezen, W. Timens, D.S. Postma, H.J. Groen, A. van den Berg (2007) Microarray amplification bias: loss of 30\% differentially expressed genes due to long probe poly(A)-tail distances. BMC Genomics 8: 277.
Bolos, V., H. Peinado, M.A. Perez-Moreno, M.F. Fraga, M. Esteller, A. Cano (2003) The transcription factor Slug represses E-cadherin expression and induces epithelial to mesenchymal transitions: a comparison with Snail and E47 repressors. J Cell Sci 116: 499-511.

Cano, A., M.A. Perez-Moreno, I. Rodrigo, A. Locascio, M.J. Blanco, M.G. del Barrio, F. Portillo, M.A. Nieto (2000) The transcription factor snail controls epithelial-mesenchymal transitions by repressing E-cadherin expression. Nat Cell Biol 2: 76-83.

Chen, H., Z. Liu, S. Gong, X. Wu, W.L. Taylor, R.W. Williams, S.G. Matta, B.M. Sharp (2011) Genome-wide gene expression profiling of nucleus accumbens neurons projecting to ventral pallidum using both microarray and transcriptome sequencing. Front Neurosci 5: 98.

Condeelis, J., R. Weissleder (2010) In vivo imaging in cancer. Cold Spring Harb Perspect Biol 2: a003848.

Davis, E.M., J.P. Trinkaus (1981) Significance of cell-to-cell contacts for the directional movement of neural crest cells within a hydrated collagen lattice. J Embryol Exp Morphol 63: 29-51.

Derby, M.A. (1982) Environmental factors affecting neural crest differentiation: melanocyte differentiation by crest cells exposed to cellfree (deoxycholate-extracted) dermal mesenchyme matrix. Cell Tissue Res 225: 379-386.

Derby, M.A., D.F. Newgreen (1982) Differentiation of avian neural crest cells in vitro: absence of a developmental bias toward melanogenesis. Cell Tissue Res 225: 365-378.

Duftner, N., J. Larkins-Ford, M. Legendre, H.A. Hofmann (2008) Efficacy of RNA amplification is dependent on sequence characteristics: implications for gene expression profiling using a cDNA microarray. Genomics 91: 108117.
Emmert-Buck, M.R., R.F. Bonner, P.D. Smith, R.F. Chuaqui, Z. Zhuang, S.R. Goldstein, R.A. Weiss, L.A. Liotta (1996) Laser capture microdissection. Science 274: 998-1001.

Erickson, C.A., K.W. Tosney, J.A. Weston (1980) Analysis of migratory behavior of neural crest and fibroblastic cells in embryonic tissues. Dev Biol 77: 142-156.

Friedl, P., S. Alexander (2011) Cancer invasion and the microenvironment: plasticity and reciprocity. Cell 147: 992-1009.

Fukumura, D., D.G. Duda, L.L. Munn, R.K. Jain (2010) Tumor microvasculature and microenvironment: novel insights through intravital imaging in pre-clinical models. Microcirculation 17: 206-225.

Gammill, L.S., J. Roffers-Agarwal (2010) Division of labor during trunk neural crest development. Dev Biol 344: 555-565.

- Giampieri, S., S. Pinner, E. Sahai (2010) Intravital imaging illuminates transforming growth factor beta signaling switches during metastasis. Cancer Res 70: 3435-3439.

Haldin, C.E., C. LaBonne (2010) SoxE factors as multifunctional neural crest regulatory factors. Int J Biochem Cell Biol 42: 441-444.

Hamburger, V., H. Hamilton (1951) A series of normal stages in the development of the chick embryo. J Morphol 88: 49-92.

Hendrix, M.J., E.A. Seftor, P.S. Meltzer, L.M. Gardner, A.R. Hess, D.A. Kirschmann, G.C. Schatteman, R.E. Seftor (2001) Expression and functional significance of VE-cadherin in aggressive human melanoma cells: role in vasculogenic mimicry. Proc Natl Acad Sci USA 98: 8018-8023.

Hendrix, M.J., E.A. Seftor, R.E. Seftor, J. Kasemeier-Kulesa, P.M. Kulesa, L.M. Postovit (2007) Reprogramming metastatic tumour cells with embryonic microenvironments. Nat Rev Cancer 7: 246-255. 
Hochedlinger, K. (2011) Embryonic stem cells: testing the germ-cell theory. Curr Biol 21: R850-R852.

Kasemeier-Kulesa, J.C., J.M. Teddy, L.M. Postovit, E.A. Seftor, R.E. Seftor, M.J. Hendrix, P.M. Kulesa (2008) Reprogramming multipotent tumor cells with the embryonic neural crest microenvironment. Dev Dyn 237: 26572666.

Kedrin, D., B. Gligorijevic, J. Wyckoff, V.V. Verkhusha, J. Condeelis, J.E. Segall, J. van Rheenen (2008) Intravital imaging of metastatic behavior through a mammary imaging window. Nat Methods 5: 1019-1021.

Kulesa, P.M., C.M. Bailey, J.C. Kasemeier-Kulesa, R. McLennan (2010) Cranial neural crest migration: new rules for an old road. Dev Biol 344: 543-554.

Kulesa, P.M., L.S. Gammill (2010) Neural crest migration: patterns, phases and signals. Dev Biol 344: 566-568.

Kulesa, P.M., J.C. Kasemeier-Kulesa, J.M. Teddy, N.V. Margaryan, E.A. Seftor, R.E. Seftor, M.J. Hendrix (2006) Reprogramming metastatic melanoma cells to assume a neural crest celllike phenotype in an embryonic microenvironment. Proc Natl Acad Sci USA 103: 3752 3757.

Kuriyama, S., R. Mayor (2008) Molecular analysis of neural crest migration. Philos Trans R Soc Lond B Biol Sci 363: 1349-1362.

Le Douarin, N.M., C. Kalcheim (1999) The Neural Crest. Cambridge, Cambridge University Press.

Levi-Montalcini, R. (1952) Effects of mouse tumor transplantation on the nervous system. Ann NY Acad Sci 55: 330-344.

Mayor, R., C. Carmona-Fontaine (2010) Keeping in touch with contact inhibition of locomotion. Trends Cell Biol 20: 319-328.

Mintz, B., K. Illmensee (1975) Normal genetically mosaic mice produced from malignant teratocarcinoma cells. Proc Natl Acad Sci USA 72: 3585-3589.

Narsinh, K.H., N. Sun, V. Sanchez-Freire, A.S. Lee, P. Almeida, S. Hu, T. Jan, K.D. Wilson, D. Leong, J. Rosenberg, M. Yao, R.C. Robbins, J.C. Wu (2011) Single cell transcriptional profiling reveals heterogeneity of human induced pluripotent stem cells. J Clin Invest 121: 1217-1221.

Nieto, M.A. (2011) The ins and outs of the epithelial to mesenchymal transition in health and disease. Annu Rev Cell Dev Biol 27: 347-376.

- Noutsias, M., M. Rohde, A. Block, K. Klippert, O. Lettau, K. Blunert, M. Hummel, U. Kuhl, H. Lehmkuhl, R. Hetzer, U. Rauch, W. Poller, M. Pauschinger, H.P. Schultheiss, H.D. Volk, K. Kotsch (2008) Preamplification techniques for real-time RT-PCR analyses of endomyocardial biopsies. BMC Mol Biol 9: 3.
Oppitz, M., C. Busch, G. Schriek, M. Metzger, L. Just, U. Drews (2007) Non-malignant migration of B16 mouse melanoma cells in the neural crest and invasive growth in the eye cup of the chick embryo. Melanoma Res 17: 17-30.

Ozsolak, F., A. Goren, M. Gymrek, M. Guttman, A. Regev, B.E. Bernstein, P.M. Milos (2010) Digital transcriptome profiling from attomole-level RNA samples. Genome Res 20: 519-525.

Paliwal, A., T. Vaissiere, Z. Herceg (2010) Quantitative detection of DNA methylation states in minute amounts of DNA from body fluids. Methods 52: 242-247.

Postovit, L.M., F.F. Costa, J.M. Bischof, E.A. Seftor, B. Wen, R.E. Seftor, A.P. Feinberg, M.B. Soares, M.J. Hendrix (2007) The commonality of plasticity underlying multipotent tumor cells and embryonic stem cells. J Cell Biochem 101: 908-917.

Ramgolam, K., J. Lauriol, C. Lalou, L. Lauden, L. Michel, P. de la Grange, A.M. Khatib, F. Aoudjit, D. Charron, C. Alcaide-Loridan, R. Al-Daccak (2011) Melanoma spheroids grown under neural crest cell conditions are highly plastic migratory/invasive tumor cells endowed with immunomodulator function. PLoS One 6: e18784.

Real, C., C. Glavieux-Pardanaud, N.M. Le Douarin, E. Dupin (2006) Clonally cultured differentiated pigment cells can dedifferentiate and generate multipotent progenitors with selfrenewing potential. Dev Biol 300: 656-669.

Ridenour, D.A., M.C. McKinney, C.M. Bailey, P.M. Kulesa (2012) CycleTrak: a novel system for the semi-automated analysis of cell cycle dynamics. Dev Biol 365: 189-195.

Sahai, E., J. Wyckoff, U. Philippar, J.E. Segall, F. Gertler, J. Condeelis (2005) Simultaneous imaging of GFP, CFP and collagen in tumors in vivo using multiphoton microscopy. BMC Biotechnol 5: 14.

-Schriek, G., M. Oppitz, C. Busch, L. Just, U. Drews (2005) Human SK-Mel 28 melanoma cells resume neural crest cell migration after transplantation into the chick embryo. Melanoma Res 15: 225-234.

-Simone, N.L., R.F. Bonner, J.W. Gillespie, M.R. Emmert-Buck, L.A. Liotta (1998) Laser-capture microdissection: opening the microscopic frontier to molecular analysis. Trends Genet 14: 272-276.

Stewart, R.A., J.S. Lee, M. Lachnit, A.T. Look, J.P. Kanki, P.D. Henion (2010) Studying peripheral sympathetic nervous system development and neuroblastoma in zebrafish. Methods Cell Biol 100: 127-152.

Takahashi, K., K. Okita, M. Nakagawa, S. Yamanaka (2007a) Induction of pluripotent stem cells from fibroblast cultures. Nat Protoc 2: 3081-3089.
Takahashi, K., K. Tanabe, M. Ohnuki, M. Narita, T. Ichisaka, K. Tomoda, S. Yamanaka (2007b) Induction of pluripotent stem cells from adult human fibroblasts by defined factors. Cell 131: 861-872.

Taneyhill, L.A., E.G. Coles, M. Bronner-Fraser (2007) Snail2 directly represses cadherin6B during epithelial-to-mesenchymal transitions of the neural crest. Development 134: 14811490.

Tang, F., C. Barbacioru, Y. Wang, E. Nordman, C. Lee, N. Xu, X. Wang, J. Bodeau, B.B. Tuch, A. Siddiqui, K. Lao, M.A. Surani (2009) mRNASeq whole-transcriptome analysis of a single cell. Nat Methods 6: 377-382.

Taniguchi, K., T. Kajiyama, H. Kambara (2009) Quantitative analysis of gene expression in a single cell by qPCR. Nat Methods 6: 503-506.

Tariq, M.A., H.J. Kim, O. Jejelowo, N. Pourmand (2011) Whole-transcriptome RNAseq analysis from minute amount of total RNA. Nucleic Acids Res 39: e120.

Topczewska, J.M., L.M. Postovit, N.V. Margaryan, A. Sam, A.R. Hess, W.W. Wheaton, B.J. Nickoloff, J. Topczewski, M.J. Hendrix (2006) Embryonic and tumorigenic pathways converge via Nodal signaling: role in melanoma aggressiveness. Nat Med 12: 925-932.

-Van Gelder, R.N., M.E. von Zastrow, A. Yool, W.C. Dement, J.D. Barchas, J.H. Eberwine (1990) Amplified RNA synthesized from limited quantities of heterogeneous cDNA. Proc Natl Acad Sci USA 87: 1663-1667.

Wyckoff, J.B., J.G. Jones, J.S. Condeelis, J.E. Segall (2000) A critical step in metastasis: in vivo analysis of intravasation at the primary tumor. Cancer Res 60: 2504-2511.

Yu, J., M.A. Vodyanik, K. Smuga-Otto, J. Antosiewicz-Bourget, J.L. Frane, S. Tian, J. Nie, G.A. Jonsdottir, V. Ruotti, R. Stewart, I.I. Slukvin, J.A. Thomson (2007) Induced pluripotent stem cell lines derived from human somatic cells. Science 318: 1917-1920.

Zhang, D., I.M. Brinas, B.J. Binder, K.A. Landman, D.F. Newgreen (2010) Neural crest regionalisation for enteric nervous system formation: implications for Hirschsprung's disease and stem cell therapy. Dev Biol 339: 280-294.

Zhang, L., C. Alt, P. Li, R.M. White, L.I. Zon, X. Wei, C.P. Lin (2012) An optical platform for cell tracking in adult zebrafish. Cytometry A 81: 176-182.

Zhao, C., X. Wang, Y. Zhao, Z. Li, S. Lin, Y. Wei, H. Yang (2011) A novel xenograft model in zebrafish for high-resolution investigating dynamics of neovascularization in tumors. PLoS One 6: e21768.

Zhou, Z.H. (2011) Atomic resolution cryo electron microscopy of macromolecular complexes. Adv Protein Chem Struct Biol 82: 1-35. 